\title{
Dose introspective design affect to the improvement of working motivation?
}

\author{
Yumena KIMURA ${ }^{1}$, Ai FUJII ${ }^{2}$, Takashi TORIIZUKA ${ }^{2}$ \\ ${ }^{1}$ Graduate School of Ochanomizu University, ${ }^{2}$ Nihon University
}

\begin{abstract}
In this study, we examined whether the design of the tool influences motivation, and what kind of design of the tool would be effective for improving work motivation if given. Especially focused on the influence of introspective design. The results showed the tools with features on the goodness of memory and feeling of use improved the motivation most, while the tools with poor usability were harder to improve motivation. From these results, it can be said the tools for improving motivation are instruments conforming to the original use and tools with introspective value, and the tool to lower motivation is a tool having a characteristic that interferes with the original use this study can say.
\end{abstract}

\section{Background and Purpose}

This study focused on the design of the tools to be used when working to address the problem of disgusting with unpleasant content work and discussed the influence on work motivation improvement.

In particular, I thought that focusing on introspective design can lead to motivation.

\section{Experimental Method}

This research presented "pen" as an example of a tool. The silhouette of the pen having various features was separately displayed on the screen and shown to the subjects, and the questionnaire was used to evaluate the influence on motivation when using them. Evaluation method was done with VAS. After plotting, the subjects of this study were interviewed why they decided so, centering on those plotted on the minus side.

Experiment 1, Experiment 2 and Experiment 3, were conducted a total of 40 subjects (average age 21.72, $\mathrm{SD}=1.16$ ). Experiment 4 was conducted with a total of 20 subjects (average age 21.85, $\mathrm{SD}=0.57$ ).

\section{3-1. Experiment 1}

As a characteristic of introspective design, this study cited two kinds, "Brand" and "Memory". In addition, in order to compare with the result with introspective design, in this study, "Feeling of use", "Interesting appearance", and "Beautiful appearance" were added, a total of 5 types were prepared. This study was conducted to clarify the effect of tools with these characteristics on motivation, and what kind of influence it gives.

\section{3-2. Results and Consideration of Experiment 1}

Memory and feeling of use improved the motivation most, and interesting looking things resulted in the hardest improvement of motivation.

It is thought that it was because the luxury impression of the brand made it difficult to use for the fact that those relating to memory were highly evaluated even in the same introspective design. Therefore, introspective design affects willingness, but from the results it can be said that the influence will differ from introspective value.

It was thought that the one specialized for the appearance gave the impression that hindered the original function of the pen. Therefore, what seems to be able to achieve the purpose intended as the tool's original purpose is considered to be easy to improve motivation. 


\section{4-1. Experiment 2}

Based on the results of Experiment 1, this study tried to clarify whether effects can be seen when other features are combined with tools with certain characteristics. Two kinds of features handled in Experiment 1 were combined and compared with the case of only one type.

\section{4-2. Results and Consideration of Experiment 2}

Characteristics related to memory and feeling of use also improved motivation by combining. Interesting appearance things have reduced motivation. Since brands with the same introspection design are harder to improve motivation than memories, differences were found in the influence on motivation even by combining the same introspective design as in Experiment 1.

The one that improves motivation in Experiment 1 improves motivation also by combining it with other features, and contrarily, the feature which is difficult to improve motivation is to make it harder to improve motivation more than the case of only original features even by adding to other features it can be said from this result.

It seems to be because brand luxurious feeling wastefully makes it difficult to use for brand combination to use feeling.

\section{5-1.Experiment 3}

Contrary to Experiment 2, in order to investigate whether the motivation would rather degrade if the evaluation of the tool is bad, the features opposite to each feature were combined with the features dealt with in Experiment 1, respectively.

\section{5-2. Results and Consideration of Experiment 3}

The opposite feeling of use and the opposite characteristic of appearance were combined with any item to reduce motivation. Memory was subject to the opposite influence.

As with the interesting appearance, the opposite feature of the appearance seems to be distracting and can not concentrate and feeling that the opposite feature of the feeling of use likewise hinders the original function and it is thought that desiring motivation has decreased even if any item is added. Regarding the things related to memory, since the influence as introspective design is large, internal elements of individuals are strong, and it is thought that the evaluation is likely to lower because the image becomes worse.

\section{6-1.Experiment 4}

This study tried to clarify the relationship between motivation and value of various introspective designs. Ten kinds of introspective designs studied were mentioned.

\section{6-2.Results and Consideration of Experiment 4}

This research, due to the difference in introspective design, the motivation and the magnitude of value were different.

Those that are deeply involved personally and those that are more likely to reflect their thoughts and preferences to individuals are considered to be introspective designs and their motivation is likely to rise. On the other hand, if the relationship between the tool and the person is indirect, as the other companies and other places recognize, the influence as an introspective design is small and the motivation is difficult to rise.

\section{Summary}

Things that motivate are those that are intrinsically valuable as those that are in line with the original use, and those that motivate in the other way are considered to have characteristics that interfere with the original use. 